\title{
Monitoring Daily Routine Anomalies in Assisted Living Environments
}

\author{
Martin Franke, Ingo Keller, and Thomas Schlegel \\ Technische Universität Dresden, Nöthnitzer Str. 46, D-01187 Dresden \\ \{firstname. lastname\}@tu-dresden.de, \\ WWW home page: http://seus.inf.tu-dresden.de
}

\begin{abstract}
In this work, we introduce our approach for detecting and presenting anomalies of daily routines in assisted living environments. The proposed concept addresses the challenges of detecting, deducing and presenting the provenance, if such anomalies occurs. With our assistive knowledge- and model-based approach, users reach higher levels of awareness about their own lives and potential irregularities. Thereby, we consider the compelling issues of detecting causes of anomalies and also presenting them without overburdening the user. A prototypical implementation substantiates our approach and introduces our forthcoming user study.
\end{abstract}

Keywords: assisted living, anomaly detection, embodied assistance, context awareness, quality of life, social intelligence, context expectation

\section{Introduction}

Demographic change causes many yet unsolved problems for future decades, such as the rise of the expenditure of healthcare or the increased burden of caregivers [3]. As one solution, several kinds of Ambient Assisted Living (AAL) systems [14] have been proposed. This kind of system aims to assist users in their domestic environment assuring their independence as long as possible and improving overall life quality [3].

One type of such systems features domestic living environments with activity recognition $[18,19]$. These systems monitor surrounding environments and user activities in order to ensure that the elderly are living safely and independently in their own homes. Additionally, they can detect a range of activities, e.g., walking, sleeping and eating. In its core, such systems with activity recognition algorithms match the current available data according to sensor values. For this purpose, supervised and unsupervised machine learning algorithms are used to build and classify training and real data.

However, beside the classification of uncorrelated activities, it becomes necessary to combine these activities to daily routines. Since a permanent interruption of routines could lead to diseases, ranging from sleeping troubles up to chronical depression [5]. Studies have shown that people, who live in regular daily rhythms, 


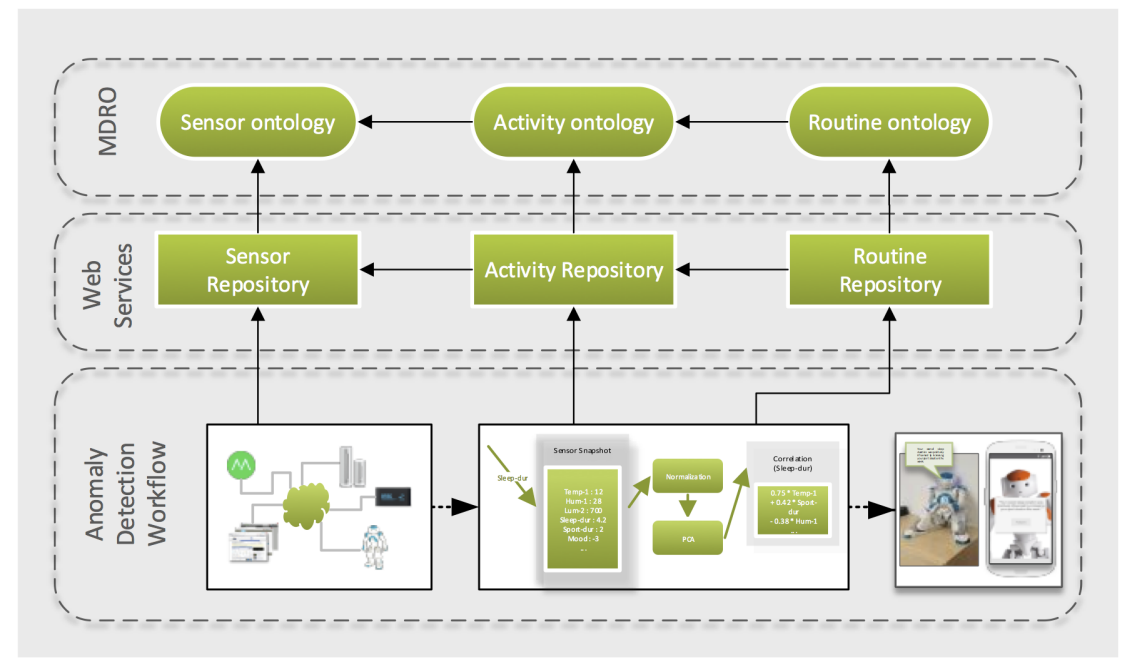

Fig. 1. Anomaly Detection Workflow

are less affected by these diseases. However, it is not always clear whether routines differ significantly from 'normal' ones. The influencing parameters are not necessarily obvious. Also, it is not properly understood how they influence the daily rhythms. This poses a challenge which we solve with our procedure. We developed a concept in our approach, which establishes awareness about such deviations and present potential provenances of it. With these findings, users, respectively caregivers, can analyse the parameters with a corresponding trend to get deeper insights about their own life.

In order to achieve the mentioned objectives, we have to answer three questions. First, how can we detect anomalies and the potential provenance proactively within our activity recognition process? Second, how can the user ask the system for trends, such as sleep or training duration, due to influencing factors? And the third aspect is how can we present the results to the user while keeping the information and system interaction intuitive? Thus, we present our solutions to tackle the mentioned problems by adapting concepts from the area of chronobiology, ambient intelligence as well as the domain of embodied assistance.

Our test environment (and system) is a domestic living environment, equipped with stationary indoor sensor devices, wearables and robotic companion for interaction purposes. In our case, NetAtmo ${ }^{1}$ weather stations, wearables, a smart phone with particular applications and a NAO robot were used (see Fig. 1). All parts will be outlined in detail in the following sections. The remaining parts of

\footnotetext{
$\overline{1}$ http://netatmo.com
} 
this article consider the following aspects: First, we discuss related work in the fields of chronobiology, assisted living environments and embodied assistance in Section 2. Then, Section 3 outlines our knowledge base and clarifies how it is applied. Afterwards, we present the corresponding awareness concept based on these model in Section 4. In Section 5, we finally draw conclusions including ideas for future work.

\section{Related Work}

We identified multiple approaches related to our work that can be classified into research of chronobiology, assisted living environments as well as approaches for embodied assistance. However, none of these approaches combine these fields, to raise awareness as necessary.

\subsection{Chronobiology}

In general cases, daily routine evolve on natural rhythms of one person. The field of chronobiology [12] examines impacts to our biological clock, such as typical sleeping cycle or times of high physical fitness.

Daily routines not only depend on personal matters, but also on exogenous and endogenous factors. These parameters influence the biological clock, as they act as orientation for the body. The most influencing example is the loss of daylight. For instance, experiments in a bunker showed that the loss of daylight extremely affects the sleep-awake rhythm. Beside the sleep irregularities, also diseases, such as manic-depressed behaviour, were discovered [17].

Another example is temperature and the exposure of light, which affect the overall activity of people. Changes in the atmospheric pressure can lead to headaches in some people and rainy conditions can reduce motivation to go outside. In contrast, spring time can lead to an extreme raise of activities until night time [12].

We use the findings of chronobiology for showing trends or factual knowledge about good life style, such as nearly eight hours of sleep or performing a 'good' amount of sport activities.

\subsection{Detecting Anomalies in Assisted Living}

The work of Yin et al. [19] illustrates an approach of activity analysis based on Hidden Markov Models (HMM). They combine multiple sensors (e.g., motion detector sensors) to HMM chains on a day-to-day basis. In contrast to other approaches, these models consist only of a correlation of sensor values to daily routines, without labelling activities. After assigning the different sensor states to HMM chains, the algorithm calculates a corridor of normal states. These reference values can be checked according to a current system state and if it is outside this corridor, the system can recognise an unusual and potentially critical situation. The advantage is that this system depends on no supervised learning. 


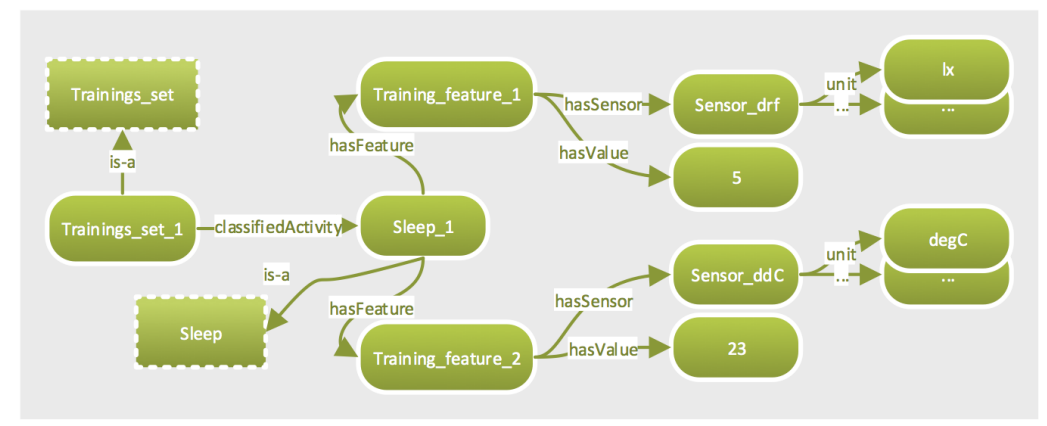

Fig. 2. Semantic Training Set (Activity Module and Sensor Module)

However, the drawback is the loss of the knowledge about the corresponding labeled activity.

The work of Botia et al. [4] uses a combination of state machines, binary sensors and rule sets for activity recognition. At the start of the system, the rule set consists of only a few rules, which are refined during runtime. Every rule corresponds to one activity and is assigned to the binary sensors, e.g. "If user is in room $A$ and no movement, then switch in state criticalSituation". The user is permanently requested to solve these critical situations by correcting the system with a new normal activity. These corrections extend the rule set, by splitting old rules into new ones, "If user is in room $A$ and is no movement and no presenceOfArmchairSensor, then switch in state criticalSituation". This system represents a good starting point to get information about deviations and the provenance. But it depends too much on specific (binary) sensors and it has been shown that rule-based systems could lead to a high overburden of the user [10].

\section{Modular Daily Routine Ontology}

The base of our activity recognition approach is a formalised, modular daily routine ontology called $M D R O$ [9]. It provides a RDF-S/OWL vocabulary for annotating data sources, such as sensor values, factual knowledge from chronobiology, recognised activities and routines. In contrast to the related works in Sect. 2.2, we produce a continuous link between all these factors from sensor values towards saved routines.

The sensor module is a semantic description of sensors to get an unified view on heterogeneous sensor values and data types [11]. This means on one hand, physical sensors in the own surrounding, such as climatic sensors. And on the other hand, also uniformed, virtual sensors, such as location or sleep duration sensors. Additionally, we formalised healthy intervals that we acquired from chronobiology [12]. With these intervals it is, subsequently, possible to show 


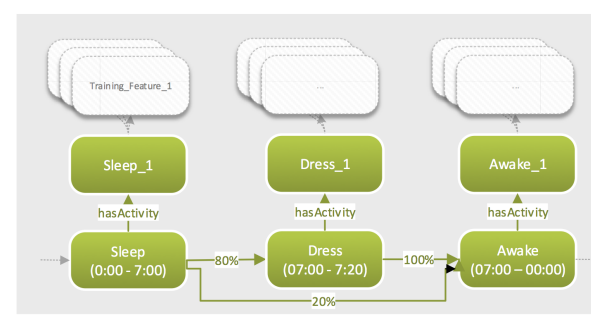

Fig. 3. Saved Routines with Links

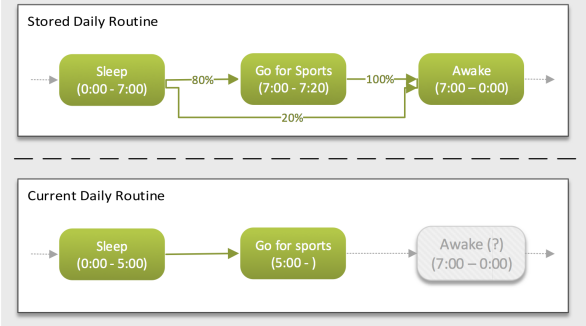

Fig. 4. Routine Deviation

the users healthy trends of their own life. A good sleep duration of 8 hours or a hint to perform sports in evening rather than in the morning can be presented and thus lead to a higher awareness of influencing parameters for well being.

The activity module holds information about possible recognised activities of daily living (ADL). As initial, but extendable set, we used the caring descriptions, mentioned as basic ADLs [8] and transferred them in an ordered semantic model of activities and sub-activities [9]. We also store the classified sensor values to corresponding activities in these module (Fig. 2). For instance, the classified activity Sleep_1 refers to the training features, which in turn link to the sensors and the training value. The effective detection, respectively recognition, of these activities is described in detail in [10].

The routine module saves a directed chain of ADLs with corresponding transition probabilities. Every routine is a chained storage of activities with daytime transitions to the next activity (Fig. 3). The time represents the sliding window for the classification, respectively the continuous stay in one activity. For instance, if our activity recognition algorithm uses sensor values between 7:00 - 7:10 to determine the current activity, this combination will be stored as instance in the routine module and will be grouped until the activity switches. As shown in the illustration, the property hasActivity refers to one classified instance and this instance, in turn, to the semantic training set.

The user module is utilised for authentication mechanisms and assigns all mentioned parts to a particular person.

In summary, the $M D R O$ models the concepts required for daily routine recognition. It is used to annotate sensor data, to formalise factual knowledge, to describe possible activities components and to save routines for users. Together, with these descriptions it is possible to give the user the awareness of anomalies.

\section{Monitoring Anomalies}

We split our approach in two awareness concepts. First, the proactive approach detects anomalies in the daily routines based on saved routines. In this procedure, the user gets information about the sensors, which have an impact on the deviation of the daily routine. 
Second, the reactive approach is for higher user awareness. It shows which sensors directly affect each other, such as sports duration and outside temperature. And also how much they are affected and if it shows a positive or negative trend.

\subsection{Proactive Detection}

During runtime, the proactive detection recognises anomalies in the daily routines, based on different time slots (e.g. week, month, quarter). Fig. 4 illustrates the simplified stored daily routine and the current routine that is determined by our Activity Recognition Algorithm [10]. The user will be notified, if the routine differs and is informed on which basis this difference was determined. For instance, if a daily routine takes an alternative route from Sleep to, e.g., Go For Sports way to early. This transition is used for finding all causes (such as reduced sleep duration in case of a to warm or to bright sleeping room). Thus the user can decide whether to adjust the daily routine to these new values once or only should be informed about the anomaly.

To detect anomalies on the technical level, we have to group the stored graph and compare it to the current routine (Fig. 5). Since our routine always starts at 0:00 and ends on 23:59, we get always the same starting and end point. Beside finding starting and end points, another issue was to compare different kinds of the same activity, if it occurs on different times. We use the same approach as the work from Yin [19] and group the activities in frames of six hours, to not overburden the user. For instance, Sleep that started between 0:00 and 6:00 will be grouped to the same sleep. In contrast the occurrence of Sleep between 22:00 and 0:00 would be a non comparable instance of the same activity class.

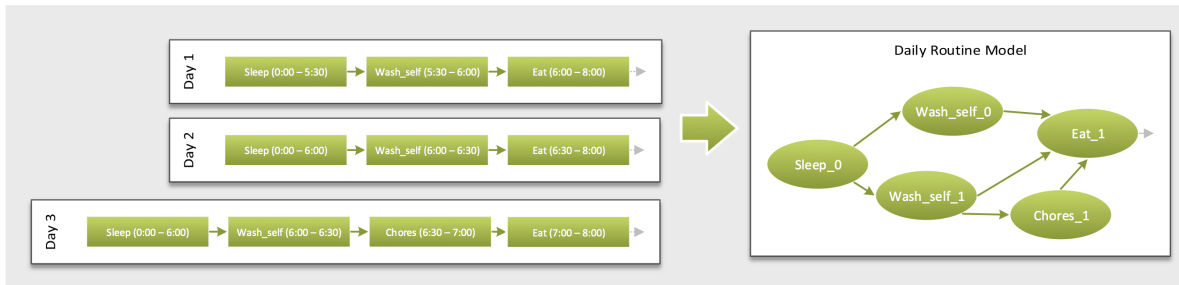

Fig. 5. Routine Transformation

After we got a comparable structure, we use an adapted VF2 [7] graph matching algorithm, which detects isomorphic structures between the stored routine models and the current daily routine. We extended therefore the VF2 to not only detect structures, but uses the semantics in the nodes. With this approach, it is 


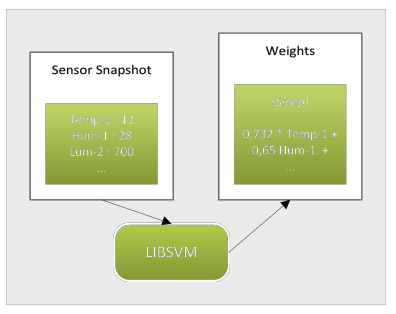

Fig. 6. Weight Comparison

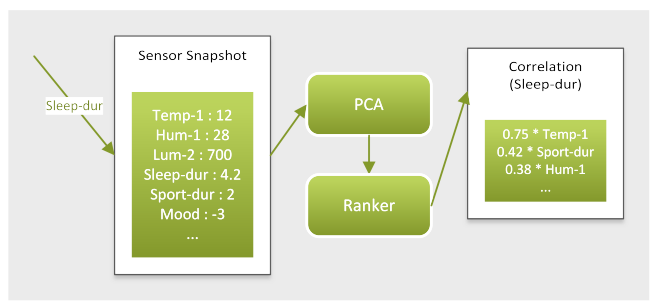

Fig. 7. Sensor Correlation Detection

possible to detect similar subroutines, even if different activities intermediates normal routines. The result is a map of all deviations between these two graphs.

After getting all points of interest, we fetch the training set, respectively the sensor values, of previous and currently classified activity from our model. The sensor values are fetched from two snapshots, see Fig. 6. The snapshot (Sensor Snapshot) holds the current sensor values, which led to the new assumed activity. With the help of LIBSVM [6, 10], we get ordered weights from the classifier and therefore the cause why the current activity not matches the stored one. These weighted sensors correlate directly with the provenance of the anomaly and are presented to the user.

\subsection{Reactive Detection}

The reactive detection is triggered by an explicit query which can be done by the user for his own dataset. The user chooses the desired feature (resp. sensor), e.g., mood or sleep duration, and will be notified about the main factors influencing the chosen feature. For instance, features, that affect the sleep duration could result in room temperature, noise and sports duration. Beside the influencing factors, we implemented rules that handle whether this change is a positive or negative one. The foundation of these assessments is derived from chronobiology and formalised in our sensor module, as previously stated. As standard, but changeable time slot, we choose a duration of one week.

The algorithm fetches the sensor values and firstly reduces the set of directly correlated sensors. This is done by the semantic (compound) description of our model. For instance, if sleep duration is the desired feature, it does not use sensors (compounds) which measure sleep directly. With this procedure, we avoid false positives such as, 'sleep duration was mainly influenced by awake time'.

To find the correlation, we use particular Factor Analysis procedure, to find the interdependencies between sensors. In detail we search for correlations with Principal Component Analysis [1] and afterwards use a Ranker to order the correlation. The potential influencing factors will be automatically sorted by this procedure.

As this procedure gives no direct correlations in terms of percentages, we use the sensors with the highest probability for further processing. By the ranked sensors, we are able to calculate the trend of sensor values. This trend is calculated by a comparison of the last week and the health interval in our model. 


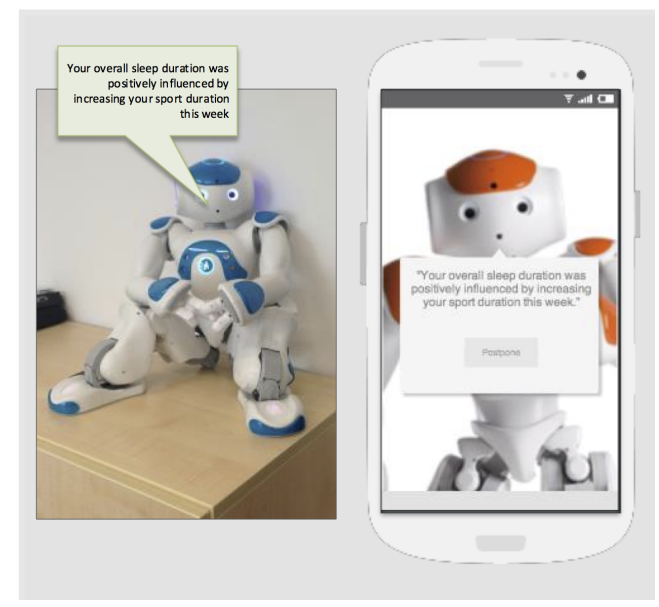

Fig. 8. Embodied Assistance

The combination of these factors is shown to the user and gives the awareness in form of: 'Your overall sleep duration was positively influenced by increasing your sport duration this week'. It is also possible to show the influencing factor's contribution rank. However, we propose that explicitly stating the contribution amount might overburden the user as the values have to be interpreted in the right way. Therefore, we show only the resulting sensors of the requested parameter, with the prior and current values.

\section{Conclusion and further work}

The increasing costs of our health care and social systems due to the changing population age structure in the western world pose a serious threat to our way of living [3]. One way to oppose these issues are domestic living environments with activity recognition $[18,19]$. These systems monitor environments and user activities, which allows, for example, the elderly to stay at their homes for as long as possible. This can only be achieved by detecting and interpreting irregularities in daily routines and by providing this information to the relevant user groups, i.e., to family members as informal caregivers and to professional caregivers. Detecting these irregularities and, especially, their causes based on sensor values remains a challenging issue. Therefore, we have presented a knowledge-assisted approach to determine the derivation and showing the potential provenance to the user and caregiver.

A relative new way to enhance assisted living environments is the use of robots within smart homes. They can assist the residents but at the same time providing the feeling of companionship as well [13]. While the robotic home companions can provide actual embodied assistance, this assistance is based on 
the data gathered by the sensors and the interaction with the robot in the smart home, e.g. Companionable [2] and Mobiserv [16].

For interacting with the system in the way we plan to use an ECA [13], once as a mobile application and as a real robot, see Fig. 8. However, this approach can be used in all situations even the NAO is not accessible, e.g., while performing outdoor activities. Additionally to a large extend the user can reuse learned interactions methods from one platform to the other, e.g., the use of the voice control. Next to the Embodied Conversational Agent the same information is displayed together with buttons to be used for touch-based input. This way, at least two different modalities are available for presenting information and getting input from the user at every time. Besides the technological aspects robot companions seem to have psychological benefits as well [15]. Specially the social companionship aspect is important which is already achieved with very simplistic interfaces. However, not in all cases in which such a social engagement might be desirable can the robot companion be physically available. Depending on the users abilities and desire, he can choose between voice based communication, text based one or a mix of both. This approach is preferable in scenarios with a high probability of people having impairments or even disabilities such as in the elderly care setting.

Currently, we are planning to conduct an in-depth user study to measure the real user confidence of our approach and also determining the thresholds of overburden such as amount of resulting sensors in correlation detection or adjusting the frequency of notifying about daily routine deviations.

\section{Acknowledgment}

This work was partly funded by the European Social Fund and the Federal State of Saxony in Germany within the projects VICCI (ESF-100098171) and SESAM (ESF-100098186).

\section{References}

[1] Charu C Aggarwal. Managing and mining sensor data. Springer, 2013.

[2] A. Badii et al. "CompanionAble: Graceful integration of mobile robot companion with a smart home environment". In: Gerontechnology 8.3 (July 2009).

[3] Ada-Helen Bayer and Leon Harper. Fixing to Stay: A National Survey on Housing and Home Modification Issues. Tech. rep. 2000.

[4] Juan A Botia, Ana Villa, and Jose Palma. "Ambient Assisted Living system for in-home monitoring of healthy independent elders". In: Expert Systems with Applications 39.9 (2012).

[5] Markus Busch, Ulfert Hapke, and Gert Mensink. Psychische Gesundheit und gesunde Lebensweise. Robert Koch-Institut, 2011.

[6] Chih-Chung Chang and Chih-Jen Lin. "LIBSVM: a library for support vector machines". In: ACM Transactions on Intelligent Systems and Technology (TIST) 2.3 (2011), p. 27. 
[7] Luigi P Cordella et al. "A (sub) graph isomorphism algorithm for matching large graphs". In: Pattern Analysis and Machine Intelligence, IEEE Transactions on 26.10 (2004), pp. 1367-1372.

[8] Anthony Fleury, Michel Vacher, and Norbert Noury. "SVM-based multimodal classification of activities of daily living in health smart homes: sensors, algorithms, and first experimental results". In: Information Technology in Biomedicine, IEEE Transactions on 14.2 (2010).

[9] Martin Franke, Ingo Keller, and Thomas Schlegel. "MDRO - A Modular Daily Routine Ontology for Assisted Living". In: Workshop on Wearable Technology and Human - Wearable Robot Interaction, (IEEE RO-MAN 2014). Aug. 2014.

[10] Martin Franke, Hagen Lehmann, and Thomas Schlegel. "Capturing and Reusing Empirical Knowledge for Activity Recognition in Assisted Living Environments". In: New frontiers of service robotics for the elderly, (IEEE RO-MAN 2014). Aug. 2014.

[11] Martin Franke, Andre Wuttig, and Thomas Schlegel. "Enabling Virtual, CloudBased Sensors in Assisted Living Environments". In: 2013 IEEE 5th International Conference on Cloud Computing Technology and Science (CloudCom). Vol. 1. Dec. 2013.

[12] Günther Hildebrandt, Maximilian Moser, and Michael Lehofer. Chronobiologie und Chronomedizin Biologische Rhythmen Medizinische Konsequenzen. Human Research, 2013.

[13] David V. Keyson et al., eds. Ambient Intelligence. Vol. 7040. Lecture Notes in Computer Science. Berlin, Heidelberg: Springer Berlin Heidelberg, 2011.

[14] Thomas Kleinberger et al. "Ambient Intelligence in Assisted Living: Enable Elderly People to Handle Future Interfaces". In: Universal Access in HumanComputer Interaction. Ambient Interaction. Ed. by Constantine Stephanidis. Lecture Notes in Computer Science 4555. Springer Berlin Heidelberg, Jan. 2007, pp. 103-112.

[15] Sean A. McGlynn et al. Therapeutic Robots for Older Adults. 2010.

[16] Maria Nani et al. MOBISERV: an integrated intelligent home environment for the provision of health, nutrition and mobility services to the elderly. 2010.

[17] Peter Spork. Das Uhrwerk der Natur: Chronobiologie-Leben mit der Zeit. Rowohlt Verlag GmbH, 2011.

[18] Caglar Tirkaz et al. "Activity recognition using a hierarchical model". In: IECON 2012-38th Annual Conference on IEEE Industrial Electronics Society. IEEE, 2012, pp. 2814-2820.

[19] GuoQing Yin and Dietmar Bruckner. "Activity analysis with hidden markov model for ambient assisted living". In: International Journal of Electronic Commerce 3.1 (2012). 